\title{
Relationship between decrease of net magnetic flux and solar major events
}

\author{
L. R. Tian ${ }^{1}$ and Y. Liu ${ }^{2}$ \\ 1 National Astronomical Observatories, Chinese Academy of Sciences, Beijing 100012, PR China \\ 2 W.W. Hansen Experimental Physics Laboratory, Stanford University, Stanford, CA 94305, USA
}

Received 13 February 2003 / Accepted 18 April 2003

\begin{abstract}
Using the full disk magnetograms taken by $S O H O / M D I$, we studied the evolution of magnetic fluxes in the lineof-sight for twelve solar active regions in the 23rd cycle, which produced strong solar activities. It is measured that the fluxes of these regions were appreciably imbalanced when they moved past the central meridian, where reliable measurements were obtained. In addition, it is found that, for seven regions for which the vector magnetic field was well observed at Huairou Solar Observing Station and thereby a vertical electric current can be derived, the vertical electric currents were out of balance. It has been found that the net fluxes of seven active regions which were associated with strong solar storms significantly decreased within 2 or 3 days prior to the major flares associated with the storms, and tended toward minimization before the onsets of the flares, suggesting a possible relationship between significant decrease of the flux imbalance of active regions and instability of large-scale magnetic fields.
\end{abstract}

Key words. Sun: activity - Sun: magnetic fields

\section{Introduction}

Imbalance of magnetic flux in the photosphere has been observed in both small and large active regions of the Sun. Livi et al. (1985) found that the flux imbalance ranges up to $30 \%$ in the ephemeral active regions; Sheeley (1966) and Stenflo (1968) reported comparably large imbalances of magnetic flux in the sunspot groups. More recently, Choudhary et al. (2002) found, using the magnetograms obtained from the National Solar Observatory at Kitt Peak, that the maximum and the median values of the flux imbalance for 137 active regions are respectively $62 \%$ and $9.5 \%$, and the active latitudinal zone in the individual hemispheres shows a flux imbalance of more than $20 \%$. Current observations further demonstrate the potential to relate the imbalance of magnetic flux of active regions with solar activity. Based on a statistical survey, Shi \& Wang (1994) showed severe imbalance of magnetic flux for the sunspots with $\delta$ magnetic structure; Tian et al. (2002) also reported that net magnetic flux in the line-of-sight could be more than $10^{21} \mathrm{Mx}$ for the super active regions in the 22nd and 23rd solar cycles, which were associated with major solar storms and/or major flares.

There are several scenarios to interpret the observed flux imbalance of active regions. In the view of observations, the reasons for the imbalance are proposed (see Gary \& Rabin 1995 and references therein) to be "(1) undetected weak field;

Send offprint requests to: L. R. Tian, e-mail: tlr@ns. bao.ac.cn
(2) merging of active-region flux with pre-existing background flux". There are also three possible physical reasons, (1) a fraction of magnetic flux of active regions connecting with remote areas, (2) the changing visibility of magnetic flux tubes (Grossman-Doerth et al. 1987), and (3) the presence of electric currents within the regions (Gary \& Rabin 1995), suggested to explain this imbalance.

YOHKOH/SXT and SOHO/EIT have observed transequatorial loops in soft X-ray and EUV wavelengths, linking active regions located on the south and north hemispheres (see, e.g. Delannée \& Aulanier 1999; Khan \& Hudson 2000; Wang et al. 2002). Wang et al. (2002) further show a good agreement of the bright transequatorial loops and the calculated magnetic field lines, suggesting that there are large-scale magnetic field lines connecting one active region and other active region(s) far away. Choudhary et al. (2002) reported that the imbalance of more than $20 \%$ was reduced to below $10 \%$ when the entire Sun is considered, indicating that a fraction of the magnetic field from the localized active regions connects with remote locations. These large-scale connections supply possible evidence that flux imbalance exists in the region of an active region.

It is suggested that flares usually occur in local areas associated with small-scale magnetic fields, while Coronal Mass Ejections (CMEs) are believed to be global phenomena associated with large-scale magnetic fields. In the view of observations, Delannée \& Aulanier (1999) and Khan \& Hudson (2000) found that the transequatorial loops linking the principal flaring 
region and some regions on the opposite side of the equator disappeared and became a main component of CMEs, proposing a picture which flares are associated with CMEs by large-scale linking. The unbalanced-flux active regions, if the large-scale linking is evidence of the flux imbalance, will connect both small-scale and large-scale magnetic fields, and thus produce the flares which are associated with large-scale responses such as CMEs.

While the reason for the flux imbalance within active regions still remains unclear (see, e.g. Gary \& Rabin 1995), it is shown that the magnitude of the flux imbalance measured in active regions more or less determines the level of solar activity (Tian et al. 2002). It is thus instructive to see if there is some relationship between evolutionary behavior of the flux imbalance and solar activity. For this, we chose 12 active regions which produced strong solar activities in the 23rd cycle to study their evolution of magnetic flux. This paper is organized as following. In Sect. 2, we will describe the observed data used, and present the solar events associated with these active regions. Evolution of magnetic flux measured and the vertical electric currents calculated will be presented in Sect. 3. We will discuss our results in Sect. 4 and draw conclusions in Sect. 5.

\section{Observation of the twelve active regions}

Information on solar events listed in Table 1 is from various sources, including the SEC Solar Event Report at http://raben@sec.noaa.gov/ftpmenu/indices/events.html and weekly report at http://www.sec.noaa.gov/ weekly/index.html, and the $S O H O / L A S C O / C M E$ catalog at http://www. cdaw.gsfc.nasa.gov/CME-list/index.html. The active regions NOAA 9077, 9632, 9684, and 9704 produced the largest proton events, and the NOAA 9415 and 9393 generated the biggest flares in the 23rd cycle.

Full disk magnetograms with 96-minute cadence taken by $S O H O / M D I$ are used to measure magnetic flux. For each active region, we sum the flux over an area which is sufficiently large to cover the whole active region, shown in Fig. 1. Considering that the MDI has a noise level of 20 Gauss (Scherrer et al. 1995), we dropped the pixels lower than 20 Gauss in our measurement of positive and negative flux. The percentage of the area below 20 Gauss is given in Table 2. We find that this area is large, up to $50-65 \%$ of the total area of the active regions. However, it will little affect net flux measurement, we will discuss the uncertainty introduced in measurement of magnetic flux and net flux in Sect. 4.

Vector magnetograms from the Huairou Solar Observing Station of the National Astronomical Observatory are used to derive the vertical electric current. A detailed description of the magnetograph and the calibration can be found elsewhere (Wang et al. 1996). The $180^{\circ}$ ambiguity for the transverse field component is resolved using a linear force-free field method (Wang \& Abramenko 2000). Vertical current density is defined as $J_{z}=\frac{1}{\mu_{0}}\left(\frac{\partial B_{y}}{\partial x}-\frac{\partial B_{x}}{\partial y}\right)$, which can be calculated by vector magnetograms in the photosphere, $B_{x}$ and $B_{y}$ are transverse components of the magnetic field. The total vertical current is a sum of the vertical current density over the area with a density higher than $6 \sigma_{J_{z}}$, where $\sigma_{J_{z}}$ is uncertainty of the current density, obtained by computing the standard deviation of the current density over points in the magnetogram where the transverse field $B_{\mathrm{t}}<100$ Gauss, the noise level of the transverse field.

\section{Imbalance of magnetic flux and vertical electric current measured}

Figure 2 shows the evolution of the positive, negative and net magnetic fluxes of the twelve regions in over interval of 4-5 days when the regions were near the central meridian. We can see that the magnetic fluxes measured in these regions are generally unbalanced before " $t_{1}$ ", the time when the major events occurred. The net fluxes of magnetic field of NOAA 9077, 9684, 9704, 9415, 9393, and 9661 significantly decreased within 2 or 3 days prior to the major flares and tended toward zero before the onset of flares (see Figs. 2a-2f), while that of NOAA 9632 shows a tendency toward zero before the flare, although the net flux could not be measured a few days before " $t_{1}$ " when the region was near the east limb (see Fig. 2g). The major event of NOAA 8100 occurred on November 6, 1997 when the region was near the west limb, and we thus cannot measure the flux on this date. The net flux decreased before this date, and showed a tendency to approach zero (see Fig. 2h). These 8 active regions, which showed similar evolutionary characteristics of magnetic flux, produced severe geomagnetic storms that lasted more than a day. One the other hand, however, the active regions NOAA 8210, 9236, 9682, and 9026, which displayed different evolutionary patterns of magnetic flux (see Figs. 2i-2l), produced much smaller (or no) radio bursts, proton events or geo-magnetic storms. The associated geo-magnetic storms last shorter than half a day. This implies that significant decrease of the flux imbalance in active regions might be a precursor of severe geo-magnetic storms.

The ratio of net and total magnetic flux measured, $\left(\left|F_{\mathrm{p}}\right|-\right.$ $\left.\left|F_{\mathrm{n}}\right|\right) /\left(\left|F_{\mathrm{p}}\right|+\left|F_{\mathrm{n}}\right|\right)$, is shown in Fig. 3. Again, for the first seven active regions (see Figs. 3a-3g), imbalance of magnetic flux appreciably decreased and tended to minimum before the major events.

Table 3 shows total vertical electric currents, calculated in areas where the electric current density $J_{z}$ is larger than $6 \sigma_{J_{z}}$ for 7 active regions when these regions were near the central meridian and the vector magnetograms were taken at HSOS. $\sigma_{J_{z}}$ denotes uncertainty of the current density, obtained by computing the standard deviation of the current density over points in the magnetogram where the transverse field $B_{\mathrm{t}}<100$ Gauss. For each fine vector magnetogram, we calculated the total positive, negative and net current above $6 \sigma_{J_{z}}$, then got a mean value from vector magnetograms available on a given day. Thus, in Table 3 , " $I_{\mathrm{p}}$ ", " $I_{\mathrm{n}}$ " and " $I_{\mathrm{t}}$ " represent the total positive, negative and net mean currents calculated, respectively. After them, the plus/minus values are mean errors, $\frac{\Sigma\left|I_{i}-I_{\text {avg }}\right|}{N-1}$, calculated on the day given. Obviously, the net currents, $I_{\mathrm{t}}$, are all larger than $10^{12} \mathrm{~A}$, indicating that total positive and negative currents are significantly unbalanced for these active regions with strong solar activities. 
Table 1. Properties of the twelve active regions and associated events in the 23rd cycle.

\begin{tabular}{|c|c|c|c|c|c|c|c|c|c|}
\hline \multirow[t]{2}{*}{ NOAA } & \multirow{2}{*}{$\begin{array}{l}\text { Date } \\
\text { Day }\end{array}$} & \multirow{2}{*}{$\begin{array}{l}\text { Location } \\
\text { Lat. CMD }\end{array}$} & \multicolumn{2}{|c|}{ Major Flares } & \multirow{2}{*}{$\begin{array}{c}\text { Proton } \\
\text { Flux }(P f u)\end{array}$} & \multirow{2}{*}{$\begin{array}{c}10.7 \mathrm{~cm} \\
\text { Flux }(S f u)\end{array}$} & \multirow[b]{2}{*}{ Index } & \multirow{2}{*}{$\begin{array}{c}\text { Kp } \\
\text { Duration (Day) }\end{array}$} & \multirow{2}{*}{$\begin{array}{c}C M E s \\
\text { Width, } V\left(\mathrm{~km} \mathrm{~s}^{-1}\right)\end{array}$} \\
\hline & & & Class & Start $(U T)$ & & & & & \\
\hline \multirow[t]{3}{*}{9077} & 11 & $N 18 E 27$ & $X 1$ & 1212 & & 1600 & & & \\
\hline & 12 & $N 16 E 31$ & $M 1.2$ & 0455 & & 43 & & & \\
\hline & & N17E27 & $X 1.9$ & 1015 & & $390, I V$ & 7 & & $56, \quad 287$ \\
\hline \multirow[t]{3}{*}{$\left(t_{1}\right)$} & 14 & $N 22 W 07$ & $X 5.7$ & 1003 & 24000 & $2600, I I, I V$ & $9^{+}$ & 1 & 360,1674 \\
\hline & & $N 20 W 08$ & M3.7 & 1344 & & 220 & & & \\
\hline & 16 & N17W38 & M1.4 & 2337 & & & & & \\
\hline \multirow{2}{*}{$\begin{array}{l}9684 \\
\left(t_{1}\right)\end{array}$} & 04 & $N 06 W 18$ & $X 1.0$ & 1603 & 31700 & $1900, I I, I V$ & 8 & $1^{+}$ & $360, \quad 1810$ \\
\hline & 05 & $N 02 W 37$ & $M 2.1$ & 0907 & & & & & \\
\hline \multirow[t]{2}{*}{9704} & 17 & $S 13 E 42$ & $M 2.8$ & 0449 & & $150, I I, I V$ & 4 & & 360,1380 \\
\hline & 22 & $S 18 W 33$ & $M 1.2$ & 1700 & & & & & \\
\hline \multirow[t]{2}{*}{$\left(t_{1}\right)$} & & $S 15 W 34$ & $M 9.9$ & 2232 & 18900 & $9700, I I, I V$ & $9^{+}$ & 1 & 360,1437 \\
\hline & 25 & & $X 1.1$ & 0945 & & & & & \\
\hline 9415 & 05 & $S 24 E 50$ & $M 5.1$ & 1657 & & 2800 & 4 & & 360,1390 \\
\hline & 06 & $S 24 E 31$ & $X 5.6$ & 1910 & & 3300 & 6 & & $360, \quad 1270$ \\
\hline & 09 & $S 21 W 04$ & $M 7.9$ & 1520 & & $1600, I I, I V$ & 3 & & 360,1192 \\
\hline$\left(t_{1}\right)$ & 10 & $S 23 W 09$ & $X 2.3$ & 0506 & 355 & $4000, I I, I V$ & 8 & 1 & 360,2411 \\
\hline & 11 & $S 22 W 27$ & $M 2.3$ & 1256 & & $540, I I, I V$ & 3 & & 360,1103 \\
\hline 9393 & 28 & N18E02 & $M 4.3$ & 1121 & & 78 & & & 360,519 \\
\hline & & $N 14 W 05$ & $M 1.5$ & 1858 & & & & & 59,258 \\
\hline & & $N 17 W 01$ & $M 1.6$ & 2218 & & 130 & & & \\
\hline & & $N 14 W 08$ & $M 2.2$ & 2325 & & 70 & & & \\
\hline & 29 & N17W04 & $M 2.1$ & 0244 & & & & & 106,526 \\
\hline$\left(t_{1}\right)$ & & $N 17 W 18$ & $X 1$ & 0957 & 35 & $4700, I V$ & $9^{+}$ & 1 & 360,942 \\
\hline & & $N 15 W 12$ & $M 2.1$ & 1129 & & & & & \\
\hline & & $N 16 W 15$ & $M 1.6$ & 1409 & & & & & 82,509 \\
\hline & & $N 16 W 13$ & $M 1.5$ & 1452 & & & & & \\
\hline & & $N 14 W 15$ & $M 1.2$ & 1520 & & & & & \\
\hline & 30 & $N 14 W 24$ & $M 2.2$ & 0511 & & 91 & & & \\
\hline 9661 & 19 & $N 16 W 18$ & $X 1.6$ & 0047 & & $1400, I I, I V$ & 3 & & 254,558 \\
\hline$\left(t_{1}\right)$ & & $N 15 W 29$ & $X 1.6$ & 1613 & 11 & $950, I I, I V$ & 7 & 2 & 360,901 \\
\hline 9632 & 23 & $S 18 E 40$ & $M 1.1$ & 1131 & & & & & \\
\hline$\left(t_{1}\right)$ & 24 & $S 16 E 23$ & $X 2.6$ & 0936 & 12900 & $7500, I I, I V$ & 6 & 0.5 & 360,2402 \\
\hline 8100 & 03 & $S 20 W 15$ & $M 1.4$ & 0903 & & $130, I I, I V$ & 3 & & 112,352 \\
\hline & & $S 19 W 20$ & $M 1.0$ & 1955 & & II & & & \\
\hline & 04 & $S 20 W 24$ & $M 1.3$ & 0127 & & & & & \\
\hline & & $S 20 W 25$ & M4.1 & 0232 & & & & & \\
\hline & & $S 14 W 33$ & $X 2.1$ & 0552 & 72 & $690, I I, I V$ & 4 & & 360,785 \\
\hline$\left(t_{1}\right)$ & 06 & $S 18 W 63$ & $X 9.4$ & 1149 & 490 & $3300, I I$ & 7 & 0.3 & 360,1556 \\
\hline 8210 & 29 & $S 18 E 20$ & $M 6.8$ & 1606 & & $100, I V$ & 4 & & 360,1374 \\
\hline$\left(t_{1}\right)$ & 02 & $S 15 W 15$ & $X 1.1$ & 1331 & 150 & $1300, I V$ & 5 & 0.5 & 360,938 \\
\hline & 03 & $S 13 W 34$ & M1.4 & 2112 & & $810, I V$ & 3 & & 360,649 \\
\hline 9236 & 23 & $N 22 W 03$ & $M 1.0$ & 2318 & & $120, I I$ & 3 & & 157,690 \\
\hline$\left(t_{1}\right)$ & 24 & N20W05 & $X 2.0$ & 0455 & 942 & $2200, I I$ & 3 & & 360,994 \\
\hline & & $N 22 W 07$ & $X 2.3$ & 1451 & & $1400, I I$ & 3 & & $360, \quad 1245$ \\
\hline & & $N 21 W 14$ & $X 1.8$ & 2143 & & 110 & & & \\
\hline & 25 & $N 18 W 24$ & M3.5 & 0906 & & 100 & & & \\
\hline & & $N 20 W 23$ & $X 1.9$ & 1833 & & $1100, I I$ & 4 & & 360,671 \\
\hline & 26 & N19W30 & $M 2.2$ & 0247 & & 60 & & & \\
\hline & & $N 18 W 38$ & $X 4.0$ & 1634 & & $700, I I$ & 6 & 0.5 & 360,980 \\
\hline 9682 & 29 & $N 12 E 26$ & M3.6 & 1056 & & $58, I V$ & & & \\
\hline & & $N 12 E 23$ & $M 1.6$ & 1130 & 26 & & & & \\
\hline & & $N 12 E 20$ & $M 1.5$ & 2349 & & & & & \\
\hline & 31 & $N 11 E 02$ & M3.2 & 0734 & & 140 & & & \\
\hline & 01 & $N 12 W 23$ & M1.1 & 2138 & & 34 & & & $47, \quad 145$ \\
\hline 9026 & 06 & $N 18 E 12$ & $X 1.1$ & 1330 & & 560 & & & \\
\hline & & & $M 7.1$ & 1356 & & & & & \\
\hline$\left(t_{1}\right)$ & & $N 20 E 18$ & $X 2.3$ & 1458 & 84 & $2300, I I, I V$ & 7 & 0.5 & 360,1119 \\
\hline & 07 & $N 23 W 03$ & $X 1.2$ & 1534 & & $200, I I, I V$ & 3 & & 360,842 \\
\hline & 10 & $N 22 W 38$ & M5.2 & 1640 & 46 & $550, I I$ & 5 & & $360, \quad 1108$ \\
\hline
\end{tabular}




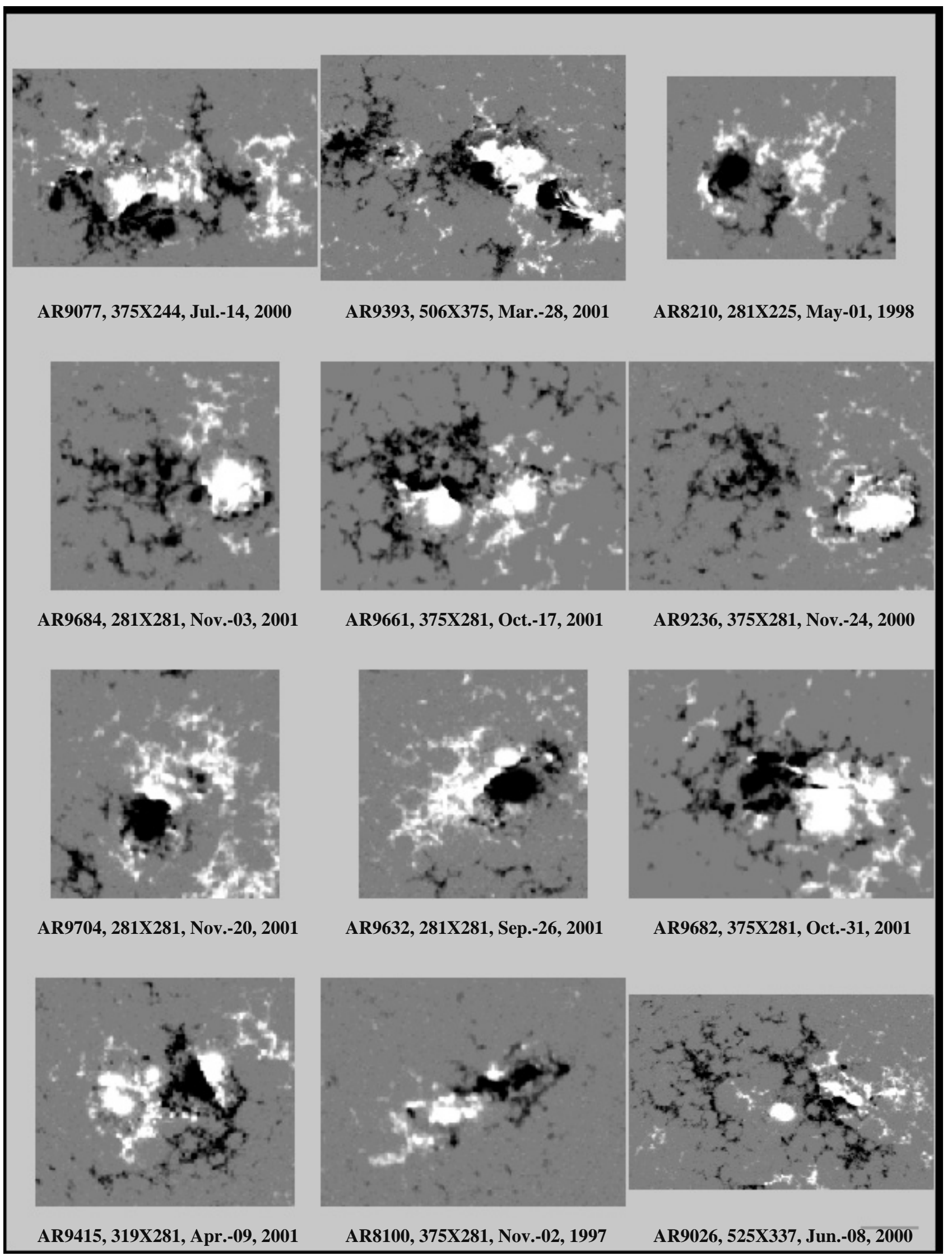

Fig. 1. Regions selected to measure the magnetic fluxes of 12 active regions. Black denotes negative polarity, white positive polarity. The unit of the area of active regions is arcsec and arcsec. 

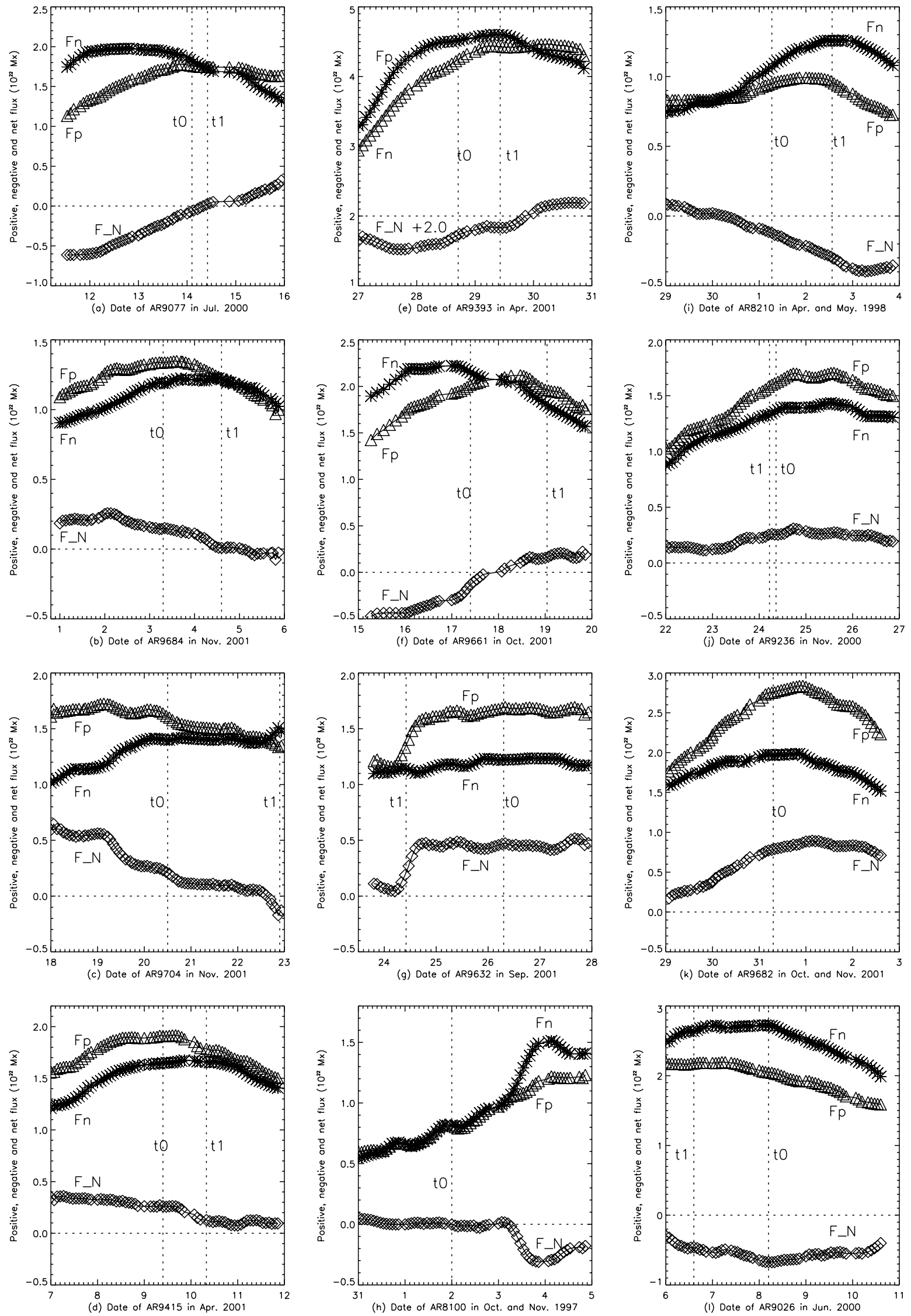

Fig. 2. The flux evolution of the twelve SARs. " $\triangle$ " $\left(F_{\mathrm{p}}\right)$, “*” $\left(F_{\mathrm{n}}\right)$ and " $\diamond "\left(F_{-N}\right)$ denote positive, negative and net magnetic flux. " $t_{0}$ " is the time when the active regions crossed the central meridian. " $t_{1}$ " is the time when the major flare occurred which is associated with severe solar storms, radio bursts, and (or) large proton events. 


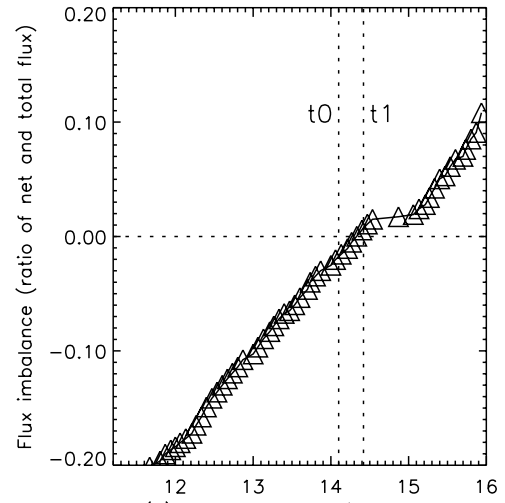

(a) Date of AR9077 in Jul. 2000

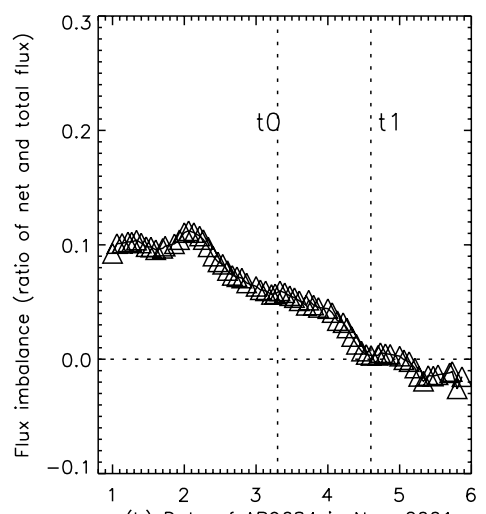

(b) Date of AR9684 in Nov. 2001
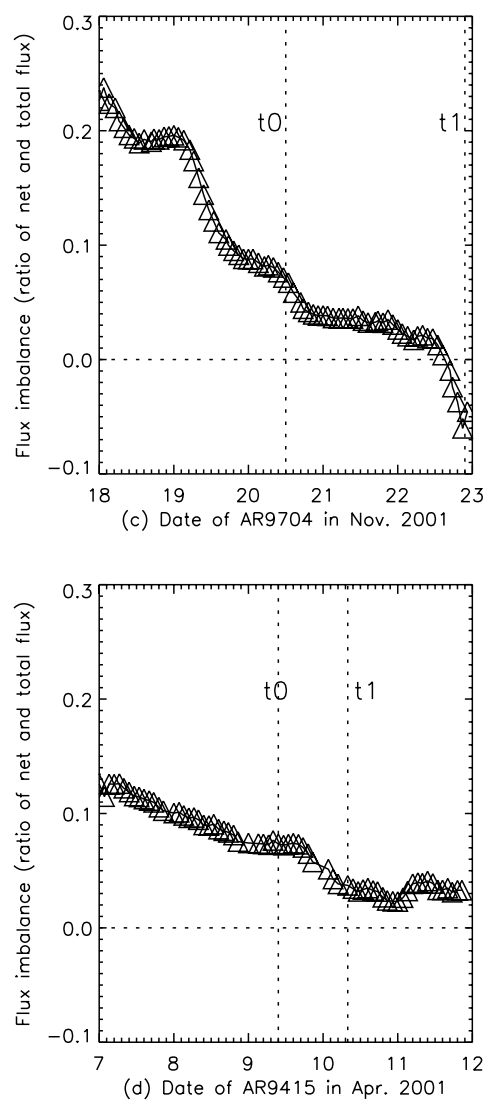

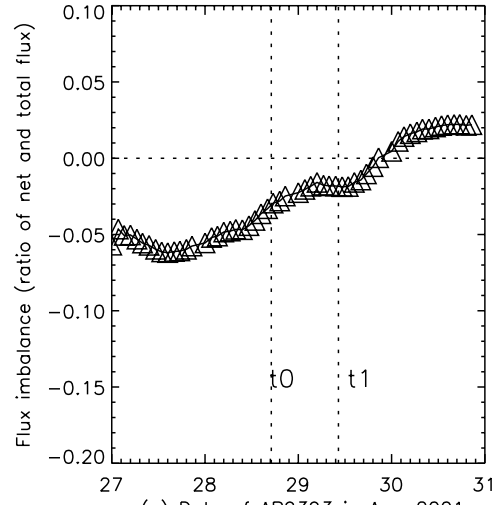

(e) Date of AR9393 in Apr. 2001
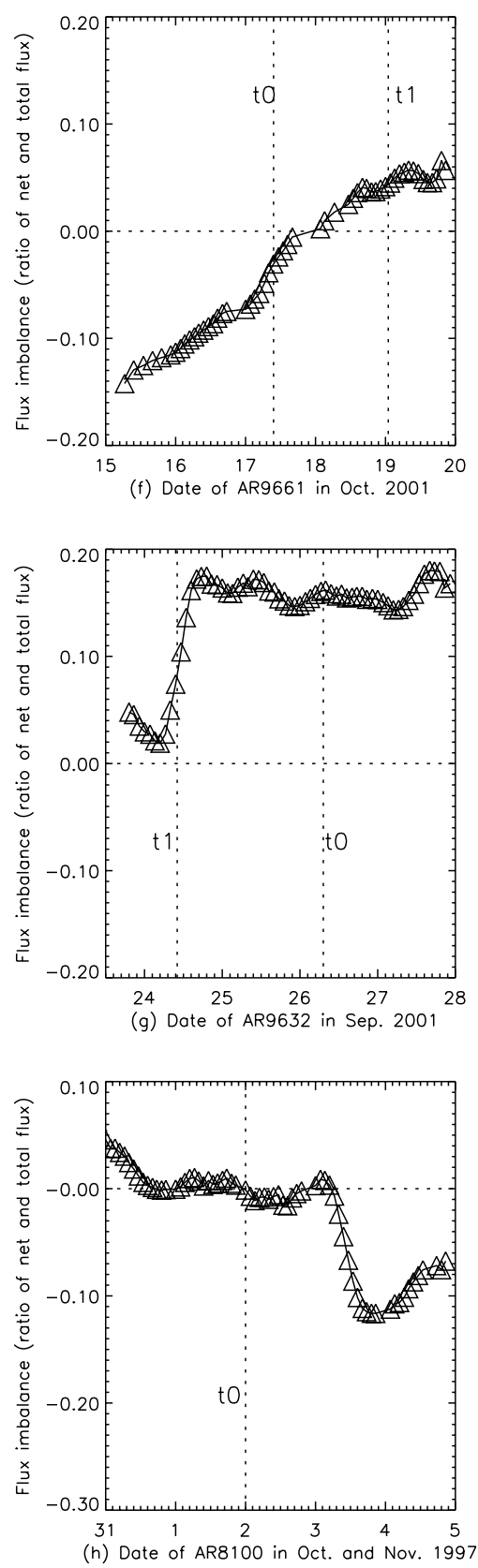
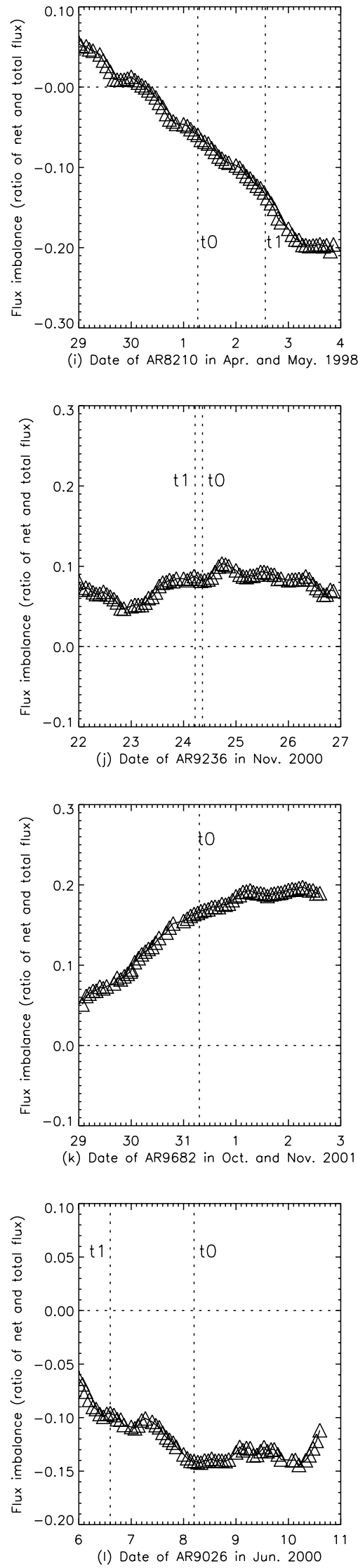

Fig. 3. The flux imbalance of the twelve SARs, indicated by ratio of the net and total magnetic flux $\left(\left(\left|F_{\mathrm{p}}\right|-\left|F_{\mathrm{n}}\right|\right) /\left(\left|F_{\mathrm{p}}\right|+\left|F_{\mathrm{n}}\right|\right)\right)$. " $t_{0}$ ” is the time when the active regions crossed the central meridian. " $t_{1}$ " is the time when the major flare occurred which is associated with severe solar storms, radio bursts and (or) large proton events. 
Table 2. Percentage of areas ( $B<20$ Gauss) to total area of active regions.

\begin{tabular}{lccccc}
\hline \hline NOAA & Total Area $\left(\right.$ arcsec $\left.^{2}\right)$ & Area $(B<20$ Gauss $)$ & NOAA & Total Area $\left(\right.$ arcsec $\left.^{2}\right)$ & Area $(B<20$ Gauss $)$ \\
\hline 9077 & $375 \times 244$ & $57 \%$ & 9632 & $281 \times 281$ & $47 \%$ \\
9684 & $281 \times 281$ & $55 \%$ & 8100 & $281 \times 225$ & $80 \%$ \\
9704 & $281 \times 281$ & $65 \%$ & 8120 & $375 \times 281$ & $51 \%$ \\
9415 & $319 \times 281$ & $53 \%$ & 9236 & $375 \times 281$ & $65 \%$ \\
9393 & $506 \times 375$ & $57 \%$ & 9682 & $375 \times 281$ & $49 \%$ \\
9661 & $375 \times 281$ & $45 \%$ & 9026 & $525 \times 337$ & $49 \%$ \\
\hline
\end{tabular}

Table 3. Mean and net current calculated in the active regions.

\begin{tabular}{lccccc}
\hline \hline NOAA & Date & $\sigma_{J_{z}}\left(\mathrm{~A} / \mathrm{m}^{2}\right)$ & $I_{\mathrm{p}}\left(10^{12} \mathrm{~A}\right)$ & $I_{\mathrm{n}}\left(10^{12} \mathrm{~A}\right)$ & $I_{t}\left(10^{12} \mathrm{~A}\right)$ \\
\hline 9077 & Jul. 13,00 & 0.0017 & $7.5 \pm 0.7$ & $5.0 \pm 0.5$ & $2.5 \pm 0.3$ \\
9684 & Nov. 03,01 & 0.0018 & $5.1 \pm 0.4$ & $3.4 \pm 0.3$ & $1.7 \pm 0.2$ \\
9704 & Nov. 20,01 & 0.0028 & $4.6 \pm 0.5$ & $7.4 \pm 0.7$ & $-3.3 \pm 0.8$ \\
9393 & Mar. 29,01 & 0.0013 & $8.4 \pm 1.1$ & $6.8 \pm 0.8$ & $1.6 \pm 0.4$ \\
9661 & Oct. 17,01 & 0.0016 & $9.8 \pm 0.6$ & $4.1 \pm 0.5$ & $5.7 \pm 0.8$ \\
9415 & Apr. 11,01* & 0.0017 & $6.1 \pm 0.1$ & $7.1 \pm 0.4$ & $-1.0 \pm 0.3$ \\
9632 & Sep. 25, 01* & 0.0037 & $4.5 \pm 0.2$ & $2.8 \pm 0.5$ & $1.7 \pm 0.4$ \\
\hline
\end{tabular}

* The dates are after time $t_{1}$, when major solar activities occurred.

\section{Discussion}

It should be noted in Fig. 2 that the longitudinal magnetic flux is a measured value in an area where magnetic fields are above the 20 Gauss contour level. The percentage of area lower than 20 Gauss is large, up to $50-65 \%$, shown in Table 2. If there is a about 20 Gauss contribution for each of these pixels, they will introduce about $4-6.5 \times 10^{21} \mathrm{Mx}$ uncertainty to the total flux. Thus, this large uncertainty would be introduced in positive/negative flux measurements. However, it almost does not affect net flux measured because the numbers of pixels with positive fields are roughly the same as that of negative fields. In Fig. 4, a histogram displays this using NOAA 9077 as an example.

It should be pointed out that net fluxes in this study are values measured for some specific assumption above 20 Gauss, possibly not real flux imbalance because the dominant reason causing the imbalance is not completely clear. We know that in sunspot penumbra the field is tilted away from the vertical, and threshold effects are also a source of artifacts in flux imbalance determination. On the other hand, projection effects may bias this study. However, in this study we have used data of active regions on 2 or 3 days about $-35^{\circ}$ to $35^{\circ}$ away from the central meridian. Generally speaking, the results in this interval are somewhat convincing.

Although the three artificial and observational biases possibly bring some systematic uncertainty to the flux imbalance, they should not be a dominant effect in our conclusion of decreasing net flux because the positive and negative flux show similar evolution in most parallel parts shown in Fig. 2, especially Figs. $2 \mathrm{~g}$, and $2 \mathrm{i}-2 \mathrm{l}$ in the west part of the disk. From this, we believe that there are possibly physical causes to

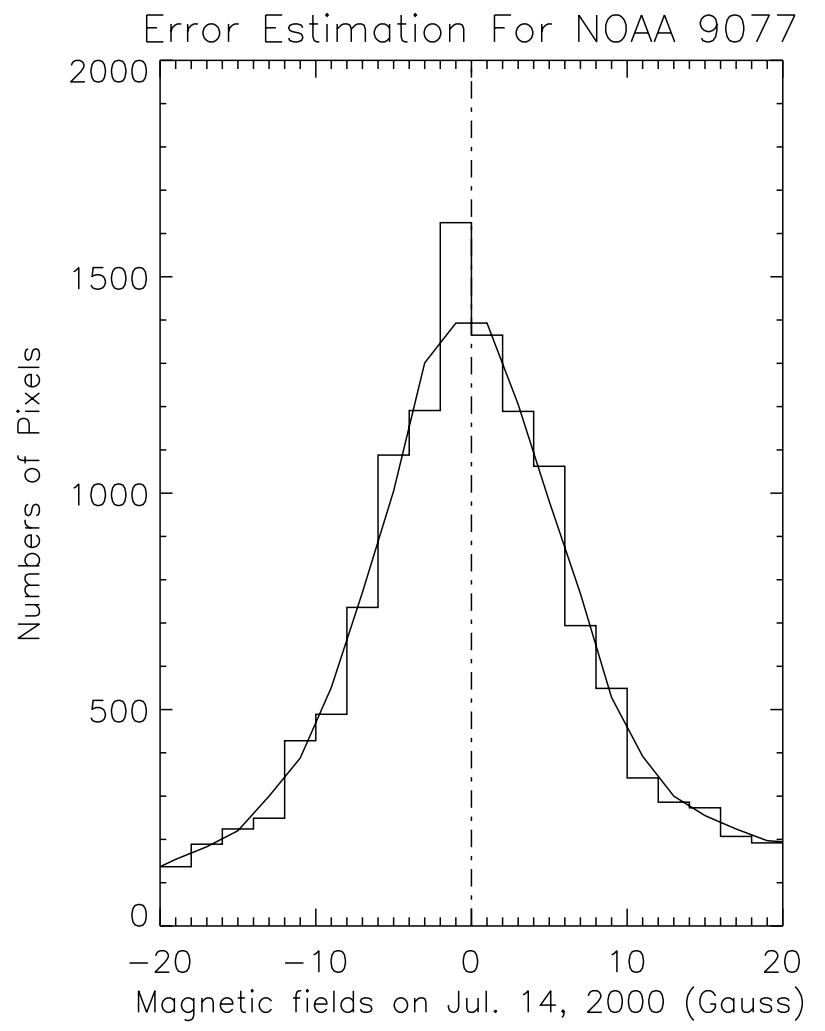

Fig. 4. Histogram of numbers of pixels with longitudinal magnetic fields lower than 20 Gauss for NOAA 9077 in area given in Fig. 1.

interpret why the net flux dropped significantly and would tend to zero before the onset of strong solar storms. However, it is indeed difficult to determine what flux is associated with the active region studied. The area selected for flux and net flux measurement may causes uncertainty, too.

Total currents in Table 3 are calculated values under the assumption of values larger than $6 \sigma_{J_{z}}$. Some observational effects and restrictions of the method used to deal with vector magnetograms would cause large uncertainties, such as $180^{\circ}$ ambiguity. Possibly, the uncertainties caused by them are larger than the $6 \sigma_{J_{z}}$ used in this paper. However, it is roughly suitable to calculate total current in an area larger than $6 \sigma_{J_{z}}$ because the total current calculated in this research is several times $10^{12} \mathrm{~A}$, very close to the typical value $\left(10^{11-12} \mathrm{~A}\right)$ of the currents derived previously by Gary et al. (1987), de La Beaujardére (1993), Wheatland (2000), and Falconer et al. (2002). 


\section{Conclusions}

We have measured magnetic flux for 12 active regions that produced strong solar events in the 23rd cycle, using the full disk magnetograms taken by $S O H O / M D I$, and studied the evolutionary behaviors of magnetic fluxes of these regions; we also calculated vertical electric currents of 7 active regions for which fine measurements of vector magnetic fields was made at HSOS. Significant imbalance of magnetic flux in these regions was measured in areas larger than 20 Gauss; the vertical currents of those 7 regions were out of balance in areas larger than $6 \sigma_{J_{z}}$. We have found that the net fluxes of seven active regions which were associated with strong solar storm, significantly decreased 2 or 3 days prior to the major flares associated, and tended toward minimization before the onset of the flares.

From YOHKOH/SXT, SOHO/EIT and other observations it is shown that some active regions are linked over a largescale (Delannée \& Aulanier 1999; Khan \& Hudson 2000; Wang et al. 2002; Choudhary et al. 2002). Furthermore, Delannée \& Aulanier (1999) and Khan \& Hudson (2000) found that the large-scale loops linking flaring regions and some remote regions disappear and become a main component of CMEs. If the large-scale connection is evidence of local flux imbalance measured, the unbalanced-flux active regions will provide a way to connect magnetic fields on a small and large scale. Thus, when small-scale fields are disturbed, such as by magnetic emergence and cancellation, the stability of the large-scale magnetic connection is affected, possibly leading to CMEs.

By this study it is suggested that the significant decrease of the net magnetic flux in individual active regions might play an key role in producing instability of large-scale magnetic fields which produces CMEs. This flux decrease generally takes two or three days, suggesting a scale of time for buildup of free energy.
Acknowledgements. L. Tian thanks Profs. Sakurai T. and Wang J. X. and Dr. Wang H. N. for their valuable suggestions and discussions. We appreciate the anonymous referee for the instructive comments and suggestions. This research is supported by NKBRSF G20000784 in China and Y. Liu was supported by NASA NAG5-3077.

\section{References}

Choudhary, D., Venkatakrishnan, P., \& Gosain, S. 2002, ApJ, 573, 851 Delannée, C., \& Aulanier, G. 1999, Sol. Phys., 190, 291

de La Beaujardére, J.-F., Canfield, R. C., \& Leka, K. D. 1993, ApJ, 411,378

Falconer, D. A., Moore, R. L., \& Gary, G. A. 2002, ApJ, 569, 1016

Gary, G. A., Moore, R. L., Hagyard, M. J., \& Haisch, B. M. 1987, ApJ, 314, 728

Gary, G. A., \& Rabin, D. 1995, Sol. Phys., 157, 185

Grossman-Doerth, U., Pahlke, K.-D., \& Schussler, M. 1987, A\&A, 176,139

Khan, J. I., \& Hudson, H. S. 2000, GeoRL, 27, 1083

Livi, S., Wang, J., \& Martin, S. F. 1985, Aust. J. Phys., 38, 855

Scherrer, P. H., Bogart, R. S., Bush, R. I., et al. 1995, Sol. Phys., 162, 129

Sheeley, N. R. 1966, ApJ, 144, 723

Shi, Z., \& Wang, J. 1994, Sol. Phys., 149, 105

Stenflo, J. O. 1968, in Structure and Development of Sol. Active Regions, ed. K. O. Kiepenheuer, IAU Symp., 35, 47

Tian, L. R., Liu, Y., \& Wang, J. X. 2002, Sol. Phys., 209, 361

Wang, J., Shi, Z., Wang, H., \& Lu, Y. 1996, ApJ, 456, 861

Wang, T. J., \& Abramenko, V. I. 2000, A\&A, 357, 1056

Wang, T., Yan, Y., Wang, J., et al. 2002, ApJ, 572, 580

Wheatland, M. S. 2000, ApJ, 532, 616 